Hakemli

\title{
CIVES, SANNITI, CONSUETUDO
}

\section{Gianluca ZARRO*}

\begin{abstract}
The essay analyzes the presence of the so-called 'paganico-vicana' structure in pre-Roman Italy and up to the fourth century B. C., coming to the consideration that this interpretation, for which the pagus should have included both isolated houses, and one or more vici inside and would have had its aggregation point in a sanctuary, which could be located in an isolated rural site or even within the vicus, must be considered outdated. However, in my opinion, the presence of the pagus or in any case of stronger settlement structures is not entirely excluded; in fact, it could be found in the archaeological evidence related to the 'barrier fortresses' established within the framework of military defensive structures.

A further aspect of research interest is the identification of 'emporium' alongside the settlement structures of the vicus; the so-called 'emporium' are to be identified in these asyla in which the negotiations took place between Romans and peregrini who were not allowed to contract through the ius civile institutes.
\end{abstract}

Keywords: paganico-vicana, pagus, vicus, emporium, asyla

\section{ÖZET}

Bu çalışmada, Roma dönemi öncesi İtalyasında M.Ö. 4. yüzyıla kadar uzanan ve "paganico-vicana" olarak adlandırılan teşkilatın varlığ 1 incelenmekte ve pagus'un hem yalıtılmış evler hem de bir veya birden fazla

Makalenin Geliş Tarihi: 28.06.2019, Makalenin Kabul Tarihi: 25.09.2019

* Doç. Dr., Suor Orsola Benincasa (Napoli) Üniversitesi, ORCID: 0000-00020171-5839 
vici'yi içermiş olması gerektiği ve taşra yerleşim yerinde veya hatta vicus dahilinde bulunabilecek toplanma merkezinin esasında bir ibadethane içinde bulunduğu yorumunun artık geçerliğinin kalmadığı düşüncesi irdelenmektedir. Bununla birlikte, yazarın görüşüne göre, pagus'un veya başka türlü güçlü bir yerleşim yeri teşkilatının varlığı tamamıyla hariç tutulmamaktadır; öyle ki askeri savunma yapıları kapsamında değerlendirilen "bariyer hisarları"na işaret eden arkeolojik bulgulardan bu durum saptanabilmektedir.

Çalışmanın ilgilendiği bir başka konu da yerleşim yeri teşkilatı olan vicus ile birlikte "emporium" un tanımlanmasıdır. "Emporium" mefhumu, ius civile'ye göre birbirleriyle akitleşmeleri yasak olan Romalılar ve peregrini (yabancılar) arasındaki hukuki işlemlerin gerçekleştiği yer olan asyla kapsamında tanımlanmaktadır.

Anahtar Kelimeler: paganico-vicana, pagus, vicus, emporium, asyla

1. I risultati degli studi sin qui condotti nell'ambito del Centro Studi sui Fondamenti del diritto antico, pertinenti al ruolo che l'ethnos sannita aveva avuto nella formazione, per usare un'espressione cara al Gabba, dell'Italia romana o romanizzata, possono ritenersi legati a filo doppio con la consuetudine: lo sono stati sia nella ricerca delle ideologie funerarie sannite, sia nell'analisi sulla formazione degli eserciti, sia nel ruolo della donna nel contesto della società sannita.

La pratica dell'inumazione, che abbiamo visto mantenersi costante dall'VIII al IV secolo a.C. presso le popolazioni sannite, specie di stirpe appenninica - qualche eccezione abbiamo creduto di confermare per l'area tirrenica, dove in virtù dei contatti con le popolazioni incineranti etrusche e greche la moderna archeologia ha riscontrato esempi di impiego della tecnica della cremazione - non può non essere riconnessa alla prassi, al ruolo che alla spontanea ripetizione di comportamenti uniformi, le gens e prima ancora le tribù sannite attribuivano al rispetto dei vincoli generazionali.

Di antica pratica consuetudinaria parla lo stesso Tito Livio in un testo famoso, Liv. $10.38 .6^{1}$, per descrivere il giuramento militare prestato nel 293

* Testo integrale della relazione tenuta presso l'Università Suor Orsola Benincasa in casa in data 22.05.2018, nell'ambito del Convegno 'Antiquitus placuit' mores, auctoritates, leges, cui è stato aggiunto un corredo di note. 
a.C. ad Aquilonia, nel corso della seconda guerra sannitica. Il rituale sacro descritto da Livio sarebbe stato tratto dai libri lintei che erano custoditi nel tempio di Giunone Moneta (arg. ex Liv 4.7.12 e 4.23.1-6); esso sarebbe stato celebrato dal vecchio sacerdote Ovius Paccius ed è significativo che lo storico patavino nel descriverlo parli di 'vetusta Samnitium religione', ma anche 'qua quondam usi maiores': "Ibi ex libro vetere linteo lecto sacrificatum sacerdote Ovio Paccio quodam, homine magno natu, qui se id sacrum petere adfirmabat ex vetusta Samnitium religione, qua quondam usi maiores eorum fuissent cum adimendae Etruscis Capuae clandestinum cepissent consilium».

1 Liv. 10, 38, 2-12: Deorum etiam adhibuerant opes, ritu quodam sacramenti vetusto velut initiatis militibus. Dilectu per omne Samnium abito nova lege, ut, qui iuniorum non convenisset ad imperatorum edictum quique iniussu abisset, eius caput Iovi sacraretur, exercitus omnis Aquiloniam est indictus. Ad quadraginta milia militum, quod roboris in Sannio erat, convenerunt. Ibi mediis fere castris locus est consaeptus cratibus pluteisque et linteis contectus, patens ducentos maxime pedes in omnis partier parties. Ibi ex libro vetere linteo tecto sacrificatum, sacerdote Ovio Paccio quodam, homine mango natu, qui se id sacrum repetere adfirmabat ex vetusta Samnitium religione, qua quondam usi maiores eorum fuissent, cum adimendae Etruscis Capuae clandestinum cepissent consilium. Sacrificio perfecto per viatorem imperator acciri iubebat nobilissimum quemque genere factisque; singuli introducebantur. Erat cum alius apparatus sacri, qui perfundere religione animum posset, tum in loco circa omni contecto arae in medio victimaeque circa caesae et circumstantes centuriones strictis gladiis. Admovebatur altaribus magis ut victima quam ut sacri particeps adigebaturque iure iurando, quae visa auditaque in eo loco essent, non enuntiaturum. Dein iurare cogebant diro quodam carmine in execrationem capitis familiaeque et stirpis composito, nisi isset in proelium, quo imperatores duxissent, et si aut ipse ex acie fugisset aut, si quem fugientem vidisset, non extemplo occidisset. Id primo quidam abnuentes iuraturos se obtruncati circa altaria sunt, iacentes deinde inter stragem victimarum documento ceteris fuere, ne abnuerent. Primoribus Samnitium ea detestatione obstrictis decem nominati ab imperatore; eis dictum, ut vir virum legerent, donec sedecim milium numerum confecissent. Ea legio linteata ab integumento consaepti, in quo sacrata nobilitas erat, appellate est; his arma insignia data et cristatae galeaeo, ut inter ceteros eminerent.

Un ulteriore escerto può rinvenirsi in: Fest. 112, 15 Lindsay: Legio Samnitum (sic) linteata appellata est, quod Samnites intrantes singuli ad aram velis linteis circumdatam non cessuros se Romano militi iuraverant. 
L'espressione qua quondam usi maiores retrodaterebbe alla presa di Capua, dunque al 423 a. C., il ritus sacramenti descritto nel passo di Livio, offrendo così una testimonianza significativa della primogenitura del suddetto rito nel settore militare, nell'ambito della koinè sannita; a me pare difficile non sostenere che la compresenza dei tre istituti, iusiurandum militare, sacertas ed il vir virum legere, seppur in presenza di una contaminazione con elementi romani - che certamente dovevano influenzare il modo di scrivere e di pensare dello storico patavino - rappresentano un evento singolare, quando non unico, nella descrizione delle pratiche dell'arruolamento degli eserciti dell'età antica.

Ancora, mi sono soffermato sulla condizione della donna nella società sannita, sottolineando che essa era ben più variegata di quello delle matronae romane, per non parlare del differente 'status' della donna romana priva di figli. L'idea di fondo di questo saggio era che la donna assumesse la veste di cacciatrice, quando non di guerriera (si parla in letteratura di 'honorary male') negli eserciti tribali sabelli per cui dopo aver manifestato la sua $\dot{\alpha} \rho \varepsilon \tau \eta ́$ all'interno della compagine sannita, forse proprio nell'attività venatoria - se non nei ludi gladiatori, che non avrebbero avuto nel Sannio carattere esiziale - assumeva un ruolo importante nella famiglia, forse munita anche di quella facoltà di operare baratti e rudimentali scambi commerciali all'interno della società sannita - tenderei infatti a sminuire la tesi, pur autorevole, che nega che i Sanniti praticassero forme di commercio ancora nel VII e nel VI secolo ${ }^{2}$.

Si può sostenere che presso i popoli Sabini, a cui i Sanniti sono tradizionalmente intrecciati, veniva praticata anche la tutela, ma questo istituto era dissimile da quello romano, giacché poteva darsi che un controllo sociale diffuso cd. 'Sozialer Druck' fosse esercitato dalla tribù stessa, senza escludere che un ruolo attivo avessero persino le stesse donne sposate, per esempio sulle 'filiae', in occasione delle nozze ${ }^{3}$.

2 G. Franciosi, Osservazioni sulle strutture sociali dei Sanniti, in Opuscoli. Scritti di Gennaro Franciosi, Napoli 2012, 633-665. Utile anche un raffronto con L. Monaco, Brevi considerazioni in tema di strutture parentali dei Sanniti, in F. M.

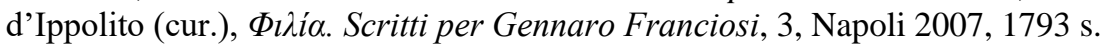

3 Parte della dottrina avanza la possibilità di una qualche riferibilità della storia di

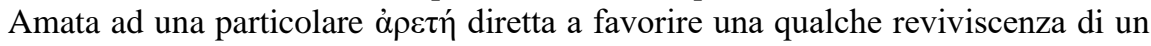
presunto diritto matriarcale; a mio avviso, la 'saga' si inserisce piuttosto piuttosto con le vicende della successione al trono. Un ulteriore riferimento ad italida 
Da questi dati emerge la natura consuetudinaria del diritto sannita, confermata dall'assenza di testimonianze scritte che documentino, seppur in via residuale, una attività normativa o almeno provvedimentale degli organi amministrativi delle tribù, fatta salva qualche residuale eccezione come le Iovile di Capua, che però sembrano appartenere ad un epoca precedente la conquista dell'urbs etrusca ed a cui deve riconoscersi un ruolo religioso, sacrale più che giuridico, diretto a confermare l'esistenza di un culto dei morti ed in particolare degli antenati.

È stato congetturato che in origine l'accezione arcaica della consuetudine corrispondesse alla legge divina. Non si intende qui riproporre le note argomentazioni del Fustel de Coulanges ${ }^{4}$ e dei primitivisti in genere tra i quali mi permetto di indicare anche il de Francisci - secondo cui la religione, il sacro costituivano fons et origo della «legge» e della vita regolata dalla comunità; gli studi più moderni ${ }^{5}$ hanno dimostrato che tale prospettiva è viziata dalla inclinazione per così dire occidentale, moderna, di presupporre una fonte precisa, dotata di autorità da cui il diritto è prodotto; per cui, non trovando nelle società antiche ed in particolare nei popoli dell'Italia arcaica una fonte precisa da cui promanasse, ci si è spinti a pensare che, agli inizi, il ius derivasse dalla dimensione del sacro, da una sorta di pace con gli dei che non era lecito turbare.

matres è rinvenibile in Ovidio, a proposito del rito dei Lupercalia. cfr. Ovid. Fast. 2.425-452.

4 N. D. Fustel De Coulanges, La cité antique. Etude sur le culte, le droit, les institutions de la Grèce et de Rome ${ }^{13,} 1980$, tr. it. 1924, 82 ss.

5 M. Bettini, Fas, in Giuristi Nati a cura di A. McClinTock, Bologna 2016, 17 ss. In particolare, sul bionomio fas et ius, senza alcuna pretesa di esaustività, per la letteratura pregressa, cfr. R. ORESTANO, Dal ius al fas. Rapporto fra diritto divino e umano in Roma dall'età primitiva all'età classica, in BIDR. 46, 1939, 244 s.; H. FUGIER, Recherches sur l'expression du sacré dans la langue latine, Paris 1963, 131; A. WALDE - J. B. HOFMANN, Lateiniscbes etymologisches Wörterbuch, I, dritte Auflage, Heidelberg 1938, 458; R. ORESTANO, Dal 'ius' al 'fas'. Rapporto fra diritto divino e umano in Roma dall'età primitiva all'età classica, in BIDR. 46, 1939, 217; ID., I fatti di normazione nell'esperienza romana arcaica, Torino 1967, 106 s.; P. NOAILlES, Du droit sacré au droit civil. Cours de droit romain approfondi 1941-1942, Paris 1949, 18; R. SANTORO, Potere ed azione nell'antico diritto romano, in Annali del Seminario giuridico dell'Università di Palermo 30, 1967, 448 ; R. SchILling, L'originalité du vocabulaire religieux latin, ora in ID., Rites, cultes, dieux de Rome, Paris 1979, 44; A. GUARINO, L'ordinamento giuridico romano ${ }^{5}$, Napoli 1990, 93 ss. 
Epperò va dato atto che una costruzione di questo tipo corrisponde a quanto è stato notato a proposito della sussistenza di una lex sacrata nei popoli italici - occorre precisare che Livio ${ }^{6}$ parla di lex sacrata - concernente la coniuratio, che in modo particolare nel descritto giuramento militare sembra saldare la fedeltà del soldato alla chiamata alle armi, in virtù della circostanza che un 'sacerdote' legga da un antico libro, ricoperto di lino, le formule che sono alla base del giuramento - tutta da decodificare è l'espressione 'ex libro vetere linteo', che appare 'prima facie' una chiara interpolazione liviana, tenendo conto sia dell'assenza di testimonianze scritte della società sannita e sabella, sia della vicenda che lega la nascita di uno ius scriptum, concernente proprio le formule pontificali, al diritto romano.

Un discorso analogo potrebbe facilmente articolarsi per uno ius fetiale ${ }^{7}$, che certamente vincolava i Sanniti, i quali non solo al momento del bellum dicere, ma anche in occasione della stipula dei trattati, appaiono condizionati da ritualità comuni a tutto il mondo antico, propedeutiche alla valida stipula di trattati e accordi ${ }^{8}$. Sappiamo, sempre da Tito Livio, che la Lega sannitica firmò un trattato con Roma (Liv. 7.19.4; Diod. 16.45.8) che possiamo annoverare nel genere del foedus aequum, che dava ad entrambi i firmatari la possibilità di far fronte a momenti di crisi. Tale trattato consentì di scongiurare in un primo momento una guerra reciproca ed era probabilmente all'origine del trattamento giuridico, in qualità di 'peregrinui' dei Sanniti, non ancora assoggettati ai Romani.

6 Liv. 10.38.6.

7 P. CATALANO, Linee del sistema sovrannazionale romano, I, Torino 1965, 30-48; A. CALORE, Forme giuridiche del 'bellum iustum', Milano 2003, 155 ss.

8 O. SACCHI, L'ager Campanus antiquus. Fattori di trasformazione e profili di storia giuridica del territorio dalla $\square \square \square \square \square \square \square \square$ arcaica alla centuriatio romana, Napoli 2004, $161 \mathrm{ss.}$

9 S. RANDAZZO, Lo statuto dello straniero e l'hospitium nel diritto romano arcaico, in Lo straniero e l'ospite. Diritto. Società. Cultura, Torino 2003, 60 ss. Occorre, infatti, mettere in risalto che il termine hostis, inizialmente, assume il ben diverso significato di 'nemico', cioè di soggetto contrapposto al civis; ad esso subentra il termine hospes, che conserva la radice di hostis, ma delinea tutt'altro concetto. L'hospitium sorge con l'accoglimento dello straniero, solennizzato con uno scambio rituale di doni, presso una famiglia romana, circostanza questa imprescindibile tanto per ragioni giuridiche che per ragioni pratiche, dal momento che l'hospes, non fruendo del ius commerci si trova nell'impossibilità concreta di acquistare beni. All'interno di queste domunculae o hospitalia lo straniero fruisce di un ausilio pratico e riveste una veste peculiare segnata dalla concessione della 
Sulla scia di questa impostazione le forme degli atti giuridici, dovevano essere sempre solenni, verbali o gestuali, prodotte da ritualità consuetudinarie.

Nelle società 'primitive', come ci testimonia anche l'esperienza romana, alla parola era affidata ogni forma di comunicazione; non è, però, alla parola in sé che in, quell'epoca, si attribuiva valore sufficiente per escarnare una volontà destinata ad incidere, modificandola, su aspetti rilevanti della realtà sociale; perché la parola avesse questa forza innovativa, essa doveva essere la parola adatta, quella che l'esperienza consolidata aveva permesso di ritenere idonea a raggiungere il risultato desiderato ${ }^{10}$; il privato che intendeva compiere un 'negozio' che richiedesse l'uso di verba, doveva, perciò, ricorrere preventivamente ai pontefici (non avendo egli strumenti di cognizione in materia), chiedere loro un responsum, doveva farsi indicare quali, nella circostanza, fossero i verba certa idonei a rendere la sua

tessera hospitatis, connotazione resa ancora più evidente dalla circostanza che l'hospitium e le fonti usano l'espressione che fanno pensare: renunciare societatem, remittere amicitia, tesseram confrigere. Lo studio delle relazioni commerciali tra i Romani ed i primi peregrini Italici appare estremamente fecondo e foriero di novità specie dal punto di vista processuale, giacché, forse, connesso con l'introduzione della legis actio per condictionem. Cfr. GIUFFrè, La "datio mutui": prospettive romane e moderne, Napoli 1989, 31 ss.

10 Nella stessa prospettiva, una certa corrente dottrinaria, invece, si era spinta, forse, troppo oltre su questa strada, giungendo ad asserire che alla radice del formalismo dei tempi antichi fossero da ravvisare credenze magiche, da cui la Roma primitiva sarebbe stata pervasa: A. HÄGERSTRÖM, Der römische Obligationsbegriff im Lichte der allgemeiner römischen Rechtsanschauung I, Uppsala-Leipzig 1927, passim; C. GIOFFREDI, Religione e diritto nella più antica esperienza romana (per la definizione del concetto di ius), in SDHI. 20, 1954, 236 s.; ID., Diritto e processo nelle antiche forme giuridiche romane, Roma 1955, 16 ss. Tracce di primitive condizioni magiche esistevano indubbiamente nel ius privatum dell'età arcaica, nonché e forse a fortiori, nel diritto pubblico, ma non tali da giustificare una preminente importanza dei rituali magici anche nel diritto della Roma delle origini: la flessibilità dei formulari rigidi e ripetitivi solo in alcune parti, la conseguente possibilità, come detto nel testo, riconosciuta ai tecnici di adeguarli alle esigenze specifiche, sono la riprova migliore che il formalismo arcaico era la manifestazione non già di un semplice primitivismo rozzo e semplificante, a sfondo magico-sacrale, ma una necessità imposta dalle particolari condizioni economico-sociali e culturali dell'ambiente in cui si viveva; da ultimo Schiavone, 'Ius'.L'invenzione del diritto in Occidente, Torino 2017, 379 e passim. 
dichiarazione un atto giuridicamente valido, pena il rischio della irrilevanza/ inesistenza del suo comportamento.

Poiché, però, la comunicazione in nessuna società, e tanto meno in quelle più antiche, era affidata soltanto alla parola, ci si serviva per le negoziazioni più impegnative, anche di contestuali tecniche del corpo, di gesti e congegni, nonché dell'uso di particolari oggetti, fatti di determinate materie ${ }^{11}$.

In altri termini, nella società arcaica, l'ambiente socio-giuridico era dominato da credenze, per lo più di carattere consuetudinario, che affidavano al costante ripetersi dei comportamenti tradizionali (i mores), all'uso di determinati oggetti (si pensi al randusculum, alla libra della mancipatio, alla festuca della vindicatio), al ricorso a certe materie (come il bronzo della mancipatio od il farro della confarreatio) la capacità di assicurare ad un atto l'approvazione sociale, in quanto 'anche' conforme ad alcune prescrizioni.

Le ragioni del fenomeno ora segnalato non possono essere identificate solo nel 'primitivismo' degli ordinamenti arcaici. L'esclusivismo di certe forme implicanti una precisa scelta di parole, di atteggiamenti e di interventi esterni, è derivato proprio dal costituirsi progressivo di una tradizione severa ed inderogabile. I rituali formali erano il prodotto della canonizzazione di comportamenti 'reali' corrispondenti a determinati usi, svolti dapprima per determinati fini e poi tipicizzati, stilizzati, irrigiditi in rappresentazioni più $o$ meno simboliche.

11 Quanto detto a proposito dell'essenza dei gesta trova una esemplificazione nella vicenda della mancipatio: l'adprehendere rem (il manus capere che dà, addirittura, nome all'atto), il pronunciare i verba aes tenens, il percuotere con il randusculum la libra, che deve essere aenea, fatta cioè di uno specifico materiale, che è qui il bronzo (cui si riconnettono specifiche virtù magiche), la consegna di questo al mancipio dans, sono tutti elementi che rendono nella mancipatio evidente che, per il simbolismo che ciascuno di essi e tutti insieme esprimono, il fatto che l'acquisto da parte del mancipio accipiens della res per la quale egli lo richiede avviene con il compimento di una serie di pratiche rituali, che esprimono, in sostanza, gli stessi gesti che secondo il normale linguaggio comportamentale accompagnano, nella pratica mercantile, lo scambio economico.

Una funzione non diversa hanno, in buona sostanza, anche le forme che consistono unicamente, come la traditio, in comportamenti.

Essenziale non è che l'autore del negozio abbia la volontà di conseguire l'effetto; essenziale è che la volontà sia resa certa e riconoscibile dall'uso di modalità idonee, in relazione alle convenzioni socio economiche diffuse. 
Tali rappresentazioni, talvolta variamente combinate, continuarono spesso ad essere impiegate anche quando si perse la comprensione del significato che avevano in origine; il che può in alcuni casi aver stimolato la credenza in un loro valore sovrannaturale imprescindibile.

In altri termini, l'esclusivismo della forma negli atti determinativi delle principali vicende dei 'negozi' più rilevanti non aveva, a mio giudizio, la stessa ragion d'essere delle liturgie religiose, anche se marginale appare, all'interno di una comunità tribale, la funzione della certezza del diritto.

2. Su questa premessa che coinvolge tanto i cives romani, quanto le comunità arcaiche e sannite in particolare, mi è sembrato possibile operare l'approfondimento di una affermazione, molto autorevole, concernente le strutture amministrative, così dette vicarie che si ipotizzano proprie dei Sanniti, anche esse a base consuetudinaria: «In proposito io non credo che allo stato, si possa affermare con qualche sicurezza una diffusa presenza in tale regione, di pagi autoctoni e, conseguentemente, per i vici del Sannio, che essi fossero originariamente entità politicamente più forti di eguale peso o meno significative dei pagi. Su questi aspetti di importanza fondamentale per valutare l'attuale dibattito intorno alle forme territoriali sannite in età preromana, mi sembra che ne sappiamo pochissimo: è bene averlo sempre presente. Ma soprattutto, lo ripeto, sia per il Sannio che per Capua i dati disponibili non ci permettono di affermare un rapporto funzionale tra vicus e pagus, nel senso che il primo, in età preromana od anche in età romana, fosse elemento costitutivo del pagus, assunto come unità politico amministrativa di base ${ }^{12} \gg$.

Intendo cioè occuparmi di quelle fonti che hanno fatto ritenere ${ }^{13}$ che $\mathrm{i}$ Sanniti vivessero in villaggi o in singole unità abitative, in fattorie.

Si è, cioè, ricostruito il paesaggio antico come se fosse caratterizzato dalla presenza di numerosi nuclei abitativi, negli immediati dintorni dei quali dovessero infittirsi le terre destinate alla coltivazione, attorno a cui si addensavano vastissime aree destinate al pascolo od al bosco.

12 L. CAPOgrossi COlOGNesi, Persistenza ed innovazione nelle strutture territoriali dell'Italia romana. L'ambiguità di una interpretazione storiografica e dei suoi modelli, Napoli 2002, 182

13 G. Tagliamonte, I Sanniti. Caudini, Irpini, Pentri, Carricini , Frentani, Milano 1996, 164 ss. 
Soprattutto Adriano La Regina ha poi ulteriormente sviluppato questa costruzione, nei primi anni settanta del secolo scorso, descrivendo in virtù di complesse analisi basate sulle evidenze funerarie - fondate principalmente sulle risultanze archeologiche dell'area sabella, su una lettura dotta della tavola dei Liguri Bebiani, nonché sui dati concernenti gli insediamenti rurali dei Vestini - un rapporto organico tra vicus e pagus, per cui quest'ultimo era la mera sommatoria di una serie di vici in esso ricompresi.

Il tema era già stato affrontato negli studi del Beloch ${ }^{14}$, concernenti la Campania antica e nelle pagine dello Schulten ${ }^{15}$, particolarmente sotto l'aspetto dell'approfondimento della questione se sussista una continuità tra le forme autoctone ed i pagi di età romana.

Queste ricerche sembrano aver trovato, per così dire, una sponda specifica all'interno della civiltà sannita, giacché come sostenuto dal Salmon ${ }^{16}$, il pagus avrebbe avuto un carattere autoctono, consuetudinario, dovendo riguardarsi ad esso come ad una sorta di sezione della più vasta unità politica tribale; anzi, ad avviso dell'eminente studioso nordamericano la «sottounità amministrativa» della tribù era costituita dal pagus, il quale, a sua volta, avrebbe potuto ricomprendere altre strutture territoriali quali i vici, gli oppida ed i castella, che per usare l'espressione dello studioso, non sembrerebbero «avere avuto una propria vita politica».

I passi addotti a sostegno erano Caes. bell. gall. 1.37 e 4.1 , cui vanno aggiunti CIL, IX, 3173 e Strab. 8.3.2. Seguendo le integrazioni operate dal Salmon sarebbe possibile aggiungere anche alcuni testi di Appiano Samn. 4.1 e dello stesso autore bell. civ. 1.51.

${ }^{14}$ BeLoch, Campanien, Geschichte und Topographie des antiken Neapel und seiner Umgebung, Berlino, Calvary 1879; $2^{\mathrm{a}}$ ed., Breslavia, Morgenstern, 1890.

15 A. SCHUlten, Die Landgemeinden im römischen Reich, in Phiolologus 35(1894); J. HEURGON, Recherches sur l'histoire, la religion et la civilisation de Capoue préromaine, Paris 1942, 182 ss. La teorizzazione romana dei vari tipi di trattato: foedus aequum, foedus iniquum, societas, societas et amicitia, amicitia non si era ancora sviluppata all'epoca del trattato del 354 a.C. Livio usa le parole socii et amicii, ma gli storici non dubitano che si tratti di una anticipazione dell'annalistica (Cass. Dio. 15.8.8). Sembra, tuttavia, che possa darsi seguito all'opinione del Salmon secondo cui si trattava di un trattato di alleanza, piuttosto che ritenere, come sembra ipotizzare il Sacchi, di un trattato diretto a mascherare sotto forma di alleanza (confederazioni militari) quelli che erano veri e propri rapporti di sottomissione.

16 E. T. SALMON, Samnium and the Samnites, Cambridge 1967, 80. 
Ancora, mette conto richiamare la prospettiva di altri studiosi, sempre di lingua americana; in particolare, intendo riferirmi agli studi del Cornell ${ }^{17}$, secondo cui «political organization of the Samnites was... simply and unsophisticated. The basic local unit was the pagus, a canton comprising one or more villages (vici) which was economically self sufficient and possessed a large measure of political autonomy», esso era governato da un meddix, secondo la nota testimonianza di Fest. 110 L. Gruppi di pagi, secondo lo studioso inglese, avrebbero, poi, in base ad uno schema sineicistico, che pare accomunare queste opzioni di ricerca, costituito «a lager tribal unit, for which the oscan term, was touto», per cui più pagi avrebbero dato luogo al touto, che coinciderebbe con il termine latino populus.

Di recente sul tema è tornato Rafael Scopacasa ${ }^{18}$, nel suo lavoro sull' 'Ancient Samnium', in cui lo studioso inglese si è spinto a sostenere un modello delle strutture territoriali del popolo sannita più variegato $\mathrm{e}$ composito, non racchiudibile nel mero binomio pagus-vicus: «Even though one must be careful not to revive outdated preconceptions about urbanization as the prime indicator of political dynamism, it is necessary to acknowledge the complexity of the settlement system in Samnium, and the existence of central settlements which acted as focal points for the construction of communities of place. In other words, the pagus-vicus model needs to be replaced by a more flexible paradigm that can account for the complexity and diversity of a local communities», sulla scorta di questi studi lo Scopacasa individua nel Monte Pallano, nei pressi del fiume Sangro uno di questi centri. Queste osservazioni suscitano un immediato richiamo ai richiamati contributi di Adriano la Regina a proposito del santuario di Pietrabbondante, che probabilmente era collegato con un centro abitativo di notevole sviluppo.

In realtà lo Scopacasa, si muove sulla scorta delle ricerche di John Loyd $^{19}$ il quale già nel 1998 metteva in dubbio il paradigna 'paganicovicano' ipotizzando insediamenti di più ampie dimensioni, i quali erano:

17 T. J. CORNELL, The Conquest of Italy, in CAH., VII.2², Cambrige 1989, 353- 356.

18 R. SCOPACASA, Ancient Samnium, Oxford 2015, 165.

19 J. LLOYD, Farming the highlands: Samnium and Arcadia in the Hellenistic and early Roman Imperial periods, in G. BARKER - A. LLOYD (cur.) Roman landscapes: archeological survey in the Mediterranean region, London 1991, 180 ss.; ID., Pentri, frentani and the beginning of urbanization (500-80 BC), in G. BARKER (cur.) A Mediterranean valley: landscape archaeology and Annales history in the Biferno valley, Leicester 1995, 181- 212. 
«large permanent settlements that incorpored different functions including residence, protection, cult, trade and decision making».

3. Qualche prima osservazione, pur con la riserva di successivi approfondimenti, si pone; per il primo gruppo di fonti in precedenza elencate - Caes. bell. gall. 1.37 e 4.1; CIL, IX, 3173 e Strab. 8.3.2.; Appiano Samn. 4.1 e dello stesso autore bell. civ. 1.51. - non ci pare che le notizie ivi raccolte potessero essere adattate alla differente realtà dell'Italia preromana.

Infatti le prime due si riferiscono alle forme organizzative proprie dei Galli e dei Germani, mentre per quanto concerne il secondo gruppo, riconducibile ad Appiano, si conferma la presenza di insediamenti vicani, di castella e di oppida fortificati, ma non ci sono elementi letterali che possono ricondurre queste unità amministrative ai pagi, che infatti non sono menzionati.

Un punto di partenza per una possibile rivisitazione di queste prospettive può essere rinvenuto nell'esegesi dettagliata offerta, da Cesare Letta $^{20}$, del passo di Festo nella classica edizione del Lindsay (L. 502 e 508), con l'integrazione generalmente accolta per la lacuna iniziale, a partire dall'edizione dell'Ursinus del 1581:

$<$ Vici appellari in>cipiunt ex agris, qui ibi villas non habent, ut Marsi aut Peligni. Sed ex vic[t] is partim habent rempublicam et ius dicitur, partim nihil eorum et tamen ibi nundinae aguntur negoti gerendi causa, et magistri vici, item magistri pagi quotannis fiunt. Altero, cum id genus aedificio $<$ rum defi>nitur, quae continentia sunt his oppidis, quae.... itineribus regionibusque distributa inter se distant, nominibusque dissimilibus discriminis causa sunt dispartita. Tertio, cum id genus aedificiorum definitur, quae in oppido privi in suo quisque loco proprio ita aedifica $<n>t$, ut in eo aedificio pervium sit, quo itinere habitatores ad suam quisque habitationem habeant accessum. Qui non dicuntur vicani, sicut hi, qui aut in oppidi vicis, aut hi, qui in agris sunt, vicani appellantur.

${ }^{20}$ C. LETTA, Nuove prospettive per lo studio di vici e pagi nell'Italia centrale appenninica, in E. CECCARONI - A. FAUSTOFERRI (eds.), Valerio Cianfarani e le culture medioadriatiche, Atti del Convegno, Chieti-Teramo, 27-29 giugno 2008, Quaderni di Archeologia d'Abruzzo 2, 2010 [2012], 65-69; S. SISANI, In pagis forisque et conciliabulis. Le strutture amministrative dei distretti rurali in Italia tra la media Repubblica e l'età municipale, Roma 2011, 559-581. Contra, E. ToDisco, I'vici' rurali nel paesaggio dell'Italia romana, Bari 2011, 57-95. 
La realtà a cui si riferisce Festo è quella degli anni che precedettero la Guerra Sociale e le grandiose trasformazioni innescate dalla municipalizzazione dell'Italia; il quadro da lui delineato, stante la ricostruzione del Letta, implicherebbe che il pagus caratterizzasse le aree di antico ager Romanus, il vicus quelle rimaste agli alleati italici, e l'ibrida mescolanza di vici e pagi quelle in cui convivevano socii italici e coloni romani, beneficiari di assegnazioni viritane.

Secondo l'interpretazione che ne dà lo studioso il significato che Festo attribuisce alla parola vici è dunque quello di un abitato rurale compatto, costituito da un agglomerato di case non sparse per la campagna, ma raggruppate in un villaggio, per cui chi vi abita alla sera non resta nei campi dove ha lavorato durante il giorno, perché la sua casa non è lì e per raggiungerla deve rientrare nel villaggio vicino da cui si è allontanato al mattino.

Ai nostri fini sembrerebbe doversi dedurre l'esclusione non solo della struttura amministrativa 'paganico-vicana' nelle popolazioni sannite così come immaginata dal Salmon e dal La Regina - in particolare per i Caudini ma addirittura che questi abbiano mai conosciuto la struttura amministrativa del pagus.

Tali affermazioni, molto autorevoli e pronunciate nell'ambito di un'interessante querelle con la Todisco ${ }^{21}$, comportano la conseguenza che il pagus non può essere considerato una realtà preromana e che le situazioni miste descritte da Festo dovrebbero spiegarsi con la compresenza nello stesso territorio di Italici portatori della tradizione insediativa dei vici e di Romani portatori di quella dei pagi.

In realtà, la voce festina si riferisce ai popoli dei Marsi e dei Peligni, dunque solo in via interpretativa (estensiva) possiamo ritenerla estesa ai popoli Sabini e Sabelli; ciò comporta la necessità di catalogare questi ultimi tra i socii, sebbene per effetto di trattati (foedera) di varia natura e con la conseguenza di attribuire a Festo l'affermazione secondo cui il pagus non avrebbe rappresentato una struttura amministrativa dell'Italia preromana, perché formatosi successivamente alle assegnazioni viritane. Deve però essere adeguatamente valutato il fatto che i Vestini, i Peligni ed i Marrucini fecero parte di un'altra lega (cd. Lega sabella), che al tempo delle guerre tra

21 E. Todisco, La percezione delle realtà rurali nell'Italia romana: $i$ vici e i pagi, in M. PANI (ed.), Epigrafia e territorio, Bari 2004, 161-184; 
Sanniti e Roma si affiancò alla Lega sannita ${ }^{22}$, sebbene è stato ritenuto ${ }^{23}$ che tale integrazione fosse indotta più da situazioni congiunturali che non per aspirare ad una integrazione di tipo federale nella Lega sannitica. Tali legami sembrano, allora, giustificare una comunanza di costumi - o magari di prassi - con i Sanniti, giacché appare difficile immaginare un'alleanza - sebbene di tipo militare - tra popoli che non condividevano neppure usanze in qualche maniera accostabili.

Questa 'interpretazione del passo festino, non appare contraddetta dalle fonti, in particolare da quel complesso dei passi che ci testimoniano che $\mathrm{i}$ Sanniti erano disseminati sul territorio vicatim, secondo quanto riportato da Livio.

Liv. 9.13.7: Samnites, ea tempestate in montibus vicatim habitant;

Liv. 10.17.2: Quid per agros inquit vagamur vicatim circumferentes bellum.

L'opinione testé descritta sembra corroborata da quanti ${ }^{24}$ hanno ritenuto che la formazione delle Leghe - mi riferisco in particolare alla Lega sannitica - non dovesse necessariamente essere intesa come formazione di un sistema federativo, prodotto del rafforzamento di vincoli tra i villaggi sottostanti.

Tuttavia, anche i più strenui assertori di questa interpretazione non possono non considerare che il contesto evolutivo della società sannita si adatta all'ipotesi della coincidenza dell'unità organizzativa conosciuta come pagus con la struttura della gens.

Ci sembra infatti possibile affermare che la comunità del villaggio, che gli studi storici hanno ritenuto di assimilare ad una fattoria, il vicus,

Salmon, Il Sannio ed i Sanniti cit. 49

${ }^{23}$ G. ZECCHINI, Il federalismo nel mondo antico, Milano 2005, 104.

${ }^{24}$ Cfr. G. ZECCHINI, Il federalismo nel mondo antico cit. 103, il cui pensiero a proposito delle organizzazioni federali di stirpi sabelle si proverà a descrivere. La tradizione greco-romana conosce un'organizzazione politica comune (Strab. 6.1.2.; C. 254 e Dion Hal. 15.8.4) dei Sanniti, dei Bretti e dei Lucani. Nel 354 a.C. i Sanniti firmarono un trattato con Roma (Liv. 7.19.4) ed è probabile che già allora avessero formato una Lega militare. Nei primi decenni del III secolo ne facevano parte i Caricini (Zon. 7.5), i Pentri (Liv. 9.31.4, Dion Hal. 17-18, 4.4), nel 210 a. C. si aggiunsero i Caudini (Liv. 22.42.1; Vell. 2.1.5) e gli Irpini (Liv. 22.42.1). 
presuppone in qualche misura un vincolo parentale, per intenderci quello che nella Roma delle origini troverà corpo nella famiglia agnatizia, mentre il pagus - pur dando per presupposto il suo carattere federativo - potrebbe attagliarsi molto bene ad una struttura gentilizia, quale ormai sembra dimostrata dalle evidenze funerarie rinvenute per esempio a Castel di Sangro o a Pietrabbondante.

Al di là di quella che, allo stato, sembra essere una mera congettura storiografica, emerge la conferma del dato che il popolo sannita non ebbe a configurarsi, sul piano politico, attraverso la forma della città-Stato ${ }^{25}$, che invece sarebbe conosciuta in altri ambienti dell'Italia antica, come quello etrusco-laziale; tale circostanza può dirsi confermata dai richiami, contenuti nelle fonti, alle tribù dei Pentri, Carricini, Frentani, Caudini, Irpini, che però non diedero mai luogo ad un ordinamento 'comune' (pur di stampo consuetudinario), come invece accade per Roma, pur generata dal sodalizio tra Tities, Ramnes e Luceres.

Cionondimeno proprio il passo dianzi trascritto sottolinea come tra $\mathrm{i}$ vici, alcuni hanno proprie istituzioni ed in essi si amministra la giustizia, altri non hanno nulla di tutto ciò; tuttavia, in essi si organizzano giorni di mercato per esercitare il commercio e come negli uni si eleggono ogni anno dei magistri vici, allo stesso modo negli altri si eleggono magistri pagi.

Ora ed al di là delle problematiche sottolineate dalla diatriba tra la Todisco ed il Letta, a me sembra rilevantissimo il richiamo a tamen ibi nundinae aguntur negoti gerendi causa, che richiama alla mente due delle categorie contenute nel celeberrimo frammento D. 50.16.19 ${ }^{26}$ attribuito a

25 Chiari i riferimenti a A. ROSENBERG, Der staat der alten Italiker. Untersuchungen über die ursprüngliche verfassung der Latiner, Osker und Etrusker, Berlin 1913, il quale sosteneva la colleganza di magistrature italiche, osco e umbro sabelliche e magistrature romane, esponeva problemi di priorità storica, lasciava chiaramente la necessità di una differente presentazione del diritto pubblico romano calato in questa nuova situazione.

26 D. 50.16.19: (Ulp. 11 ad ed.): Labeo libro primo praetoris urbani definit, quod quaedam "agantur", quaedam "gerantur", quaedam "contrahantur": et actum quidem generale verbum esse, sive verbis sive re quid agatur, ut in stipulatione vel numeratione: contractum autem ultro citroque obligationem, quod Graeci sunallagma vocant, veluti emptionem venditionem, locationem conductionem, societatem: gestum rem significare sine verbis factam. Senza alcuna pretesa di esaustività e dando la preferenza ai commenti di D. 50.16.19, si ricordano i lavori di autorevole dottrina: B. AlBANESE, 'Agere', 'gerere', 'contrahere' in D. 
Labeone, ma che mi pare conservare il pensiero del giurista severiano Ulpiano.

Infatti nella frase festina si parla di 'nundinae aguntur', ma soprattutto di 'negoti gerendi causa', il che suggerisce che si era in presenza di quei famosi empori ${ }^{27}$ in cui il popolo di Roma esercitava transazioni commerciali

50.16.19. Congetture su una definizione di Labeone, in SDHI. 38, 1972, 189 ss.; R. SANTORO, Il contratto nel pensiero di Labeone, in AUPA. 37, 1983, 5 ss. La tesi del Santoro, per la sua impostazione originale e per l'impianto delle sue argomentazioni, è stata ampiamente recensita: P. VoCI, Rec. a Santoro, in Iura 34, 1983, 124 ss.; A. GUARINO, Il contratto secondo Labeone, in Labeo 29, 1983, 329 ss.; A. BURDESE, Ancora sul contratto nel pensiero di Labeone (a proposito di uno scritto di R. Santoro), in SDHI. 51, 1983, 458 ss. Dello stesso autore: R. SANTORO, Riflessioni sul contratto nel pensiero di Labeone, in Studi Arena 5, 1981; ID., La causa nelle convenzioni atipiche, in Causa e contratto nella prospettiva storico comparatistica. II Congresso internazionale ARISTEC, Palermo-Trapani 1995. In tempi più recenti, si vedano i contributi di C.A. CAnnatA, Labeone, Aristone e il sinallagma, in Iura, 58, 2010, 33 ss.; E. SCIANDRELLO, Studi sul contratto estimatorio e sulla permuta nel diritto romano, Trento 2011, 33 ss.; Id., 'Actio de aestimato' e tipicità dei mezzi processuali. Riflessioni su Ulp. 32 'ad ed.' D. 19.3.1 pr., in L. GAROFALO (a cura di), 'Actio in rem'e 'actio in personam'. In ricordo di Mario Talamanca, II, Padova, 2011, 959 ss. Da ultimo per una differente interpretazione che approfondisce nel testo ritenuto di matrice ulpianea - proprio il lemma gestum ci sia consentito un richiamo a G. ZARro, Aspetti dell'autonomia dei privati. Dalla fides ai nova negotia, Napoli 2015, 94, 165 e ss.

27 A tutt'oggi fondamentale è il contributo di V. MAROTTA, Tutela dello scambio $e$ commerci mediterranei in età arcaica e repubblicana, in Ostraka. Rivista di antichità 5, 1996, 64, cui si rinvia anche per l'ulteriore bibliografia. L. CAPOGROSSI COLOGNESI, 'Ius commerci' 'conubium' 'civitas sine suffragio'. Le origine del diritto internazionale privato e la romanizzazione delle comunità latino-campane, in L. CAPOGrossi COLOGNESI-A. Corbino-L. LABrunA-B. SANTAluCIA, Le strade del potere, Catania 1994, 3 ss. In particolare, resta da sottolineare che nel quadro dei rapporti intergentilizi, i commerci tendevano a concentrarsi in spazi particolari, cioè all'interno dei santuari emporici, separati anche geograficamente dalle vere e proprie aree urbane, in asyla, posti sotto la propensione di un dio a presidio dell'incolumità personale dei mercanti che ivi convenivano e della sicurezza dei loro traffici. In questo senso e con riguardo alla civiltà sabella possono essere ripercorse le leggendarie vicende di Eracle in Italia. L'eroe, secondo la tradizione rivendica sempre il diritto alla giustizia per gli stranieri e mette brutalmente fine agli attentati contro l'inviolabilità delle vergini e dei boschi sacri. Il suo culto era ampiamente praticato presso i popoli de quibus. 
con i popoli stranieri - i peregrini cui accennavamo - e che questo tipo di contrattazione avveniva nelle forme dell' 'actum', nonché della gestione del negozio (gestum), che sottintende gli atti librali.

Non è possibile, infatti, ritenere che la terminologia festina se poteva presentare incertezze per quanto riguardava la descrizione delle strutture insediative sannite - cioè il vicus o il pagus - fosse imprecisa nell'impiego delle categorie romane. Pertanto, all'interno del vicus e con la garanzia del magister vicus sembra si attuassero altrettante zone di extraterritorialità in cui, sempre su base consuetudinaria, si badi, il presupposto della cittadinanza romana - categoria imprescindibile per l'impiego del ius civile- era sospeso applicandosi una sorta di diritto delle genti (italiche) fortemente contaminato da elementi, per così dire, di romanità.

Una situazione in parte differente si registra dopo il 423 a.C., nell'ambito della conquista di Capua da parte dei Sanniti.

Gli elementi di novità dipendono dalla circostanza che Capua aveva assunto la fisionomia della città-stato ${ }^{28}$, ossia di una città eletta a centro di interesse di una delle zone più fertili della penisola.

La conquista sannita può essere interpretata come attuata nell'ambito di un vero e proprio processo di riorganizzazione del territorio, ben anteriore alla romanizzazione campana. Tale conquista sembra essere il frutto di un processo 'quasi rituale' di integrazione tra elementi autoctoni, cioè quelli dei Campani originari, ossia gli Osci/Opici, l'elemento sannita - che secondo le fonti (in particolare Dionigi, cui fa da eco Livio ${ }^{29}$ ) sarebbero stati introdotti progressivamente nella società campana, sicché la conquista di Capua sarebbe stata solo l'atto conclusivo di un procedimento ben più lungo e, forse, meno cruento - ed infine un elemento allogeno, quello offerto dagli etruschi campani, già perfettamente integrati a partire dal V secolo.

Ciò che si vuole suggerire, pur con ogni cautela è che un carattere peculiare dei commerci mediterranei tra orientalizzante antico e l'età arcaica è il loro accadimento entro i santuari emporici, cioè in spazi delimitati entro i centri urbani limitrofi (il vicus), luoghi di incontro e di scambio con gli stranieri in cui avvenivano transazioni che Festo descrive come gerendi causa, espressamente ammettendo 'negozi commerciali' con i popoli dei Marsi e dei Peligni e, per conto mio, anche dei Sanniti e dei Sabelli.

28 Diod. 12.31.1.

${ }^{29}$ Liv. 4.37.2. 
Forse non sbaglia il Sacchi $^{30}$ quando si spinge ad ipotizzare che la Capua sannitica «seppe metabolizzare la civiltà, le tecniche (idrauliche, agrarie, metallurgiche) etrusche [ed osche] sancendo il passaggio in campo sociale e politico da forme tribali e cantonali ad insediamenti di tipo urbano ed a strutture organizzative di tipo federale». Siamo dinanzi ad una vera e propria città-stato che se certo non può rappresentare il territorio pentrofrentano ecc, assume, all'interno di esso, il carattere dell'autonomia.

È, però, significativo, seppur solo sul piano della sola induzione evoluzionistica, che ancora una volta si adotti come metro di tali accadimenti un processo federativo.

\section{Considerazioni conclusive}

Cercando di tirare le fila di questa relazione, ho voluto porre in evidenza le interconnessiomi giuridico-istituzionali delle strutture territoriali dei Sanniti.

Oltre alla matrice consuetudinaria o se si vuole prasseologica - è da escludere, per l'ethnos sannita, una elaborazione così complessa come lo ius moribus receptum - è emersa la presenza di un sistema differenziato di insediamenti, che appare ancora una volta accomunare i Sanniti con altre popolazioni Italiche di origine preromana, giacché tanto per gli uni quanto per gli altri sembrano configurarsi le forme abitative dei villagi (vici), dei centri fortificati (oppida) o di strutture abitative sparse nella campagna.

Allo stato non siamo in grado di testimoniare la presenza del pagus, la cui esistenza sembra da escludere non solo in virtù dei rilievi materiali, ma anche se si opera una attenta analisi delle fonti in nostro possesso; in conseguenza di queste interpretazioni sembra da ritenersi superata, la tradizionale struttura cd paganico-vicana, per la quale il pagus avrebbe dovuto comprendere al suo interno sia case isolate sia uno o più vici ed avrebbe avuto il suo punto di aggregazione in un santuario, che poteva essere ubicato in un sito rurale isolato o anche all'interno del vicus.

Tuttavia, a mio avviso, la presenza del pagus o comunque di strutture insediative più forti non è interamente da escludere; infatti essa oltre a possibili testimonianze archeologiche legate alle 'fortezze di sbarramento' costituite nell'ambito di strutture difensive militari, appare legata ad altre

${ }^{30}$ O. SACCHI, L'ager Campanus antiquus. cit. 161 ss. 
riflessioni dipendenti dall'induzione evoluzionistica e dalla struttura sineicistica che necessariamente emerge dal passaggio dalle strutture tribali a quelle gentilizie.

Un ulteriore aspetto di interesse della ricerca è l'individuazione di empori accanto alle strutture insediative del vicus, la cui precisa identificazione può forse ricondursi alla presenza di santuari, in cui i mercanti invocavano la clemenza di una qualche divinità favorevole al buon esito degli scambi. Non è impossibile vedere in ciò un richiamo alla fides, alla sua complessa evoluzione.

Al di là di congetture ancora da approfondire sembra però da scongiurare, alla luce degli studi che si vanno compiendo, l'immagine dei Sanniti, come quella di un popolo selvaggiamente guerriero privo di istituzioni e regole di stampo giuridico, dominato dall'uso della forza. Sia le indagini sul ruolo della famiglia patriarcale, sia gli studi sulle strutture insediative territoriali, ci restituiscono, invece, l'immagine di un popolo che se non riuscì a raggiungere una piena unità, per così dire politica, aveva prassi e tradizioni ben radicate che ne assicurarono lo sviluppo e ne fecero il grande antagonista di Roma, ben più insidioso di ogni altra popolazione Italica. 


\section{Bibliografia}

A. BURDESE, Ancora sul contratto nel pensiero di Labeone (a proposito di uno scritto di R. Santoro), in SDHI. 51, 1983.

A. CALORE, Forme giuridiche del 'bellum iustum', Milano 2003.

A. GUARINO, Il contratto secondo Labeone, in Labeo 29, 1983.

A. GUARINO, L'ordinamento giuridico romano ${ }^{5}$, Napoli 1990.

A. HÄGERSTRÖM, Der römische Obligationsbegriff im Lichte der allgemeiner römischen Rechtsanschauung I, Uppsala-Leipzig 1927.

A. ROSENBERG, Der staat der alten Italiker. Untersuchungen über die ursprüngliche verfassung der Latiner, Osker und Etrusker, Berlin 1913.

A. Schiavone, 'Ius'.L'invenzione del diritto in Occidente, Torino 2017.

A. SCHUlten, Die Landgemeinden im römischen Reich, in Phiolologus 35 (1894).

A. WALDE - J. B. HoFMANN, Lateiniscbes etymologisches Wörterbuch, I, dritte Auflage, Heidelberg 1938.

B. Albanese, 'Agere', 'gerere', 'contrahere' in D. 50.16.19. Congetture su una definizione di Labeone, in SDHI. 38, 1972.

C. A. CAnnAtA, Labeone, Aristone e il sinallagma, in Iura, 58, 2010.

C. GIOFFREDI, Religione e diritto nella più antica esperienza romana (per la definizione del concetto di ius), in SDHI. 20, 1954.

C. GIOFFREDI, Diritto e processo nelle antiche forme giuridiche romane, Roma 1955.

C. LETTA, Nuove prospettive per lo studio di vici e pagi nell'Italia centrale appenninica, in E. CECCARONI - A. FAUSTOFERRI (eds.), Valerio Cianfarani e le culture medioadriatiche, Atti del Convegno, Chieti-Teramo, 27-29 giugno 2008, Quaderni di Archeologia d'Abruzzo 2, 2010 [2012].

E. SCIANDRELlO, Studi sul contratto estimatorio e sulla permuta nel diritto romano, Trento 2011.

E. SCIANDRELlO, 'Actio de aestimato' e tipicità dei mezzi processuali. Riflessioni su Ulp. 32 'ad ed.' D. 19.3.1 pr., in L. GAROFAlo (a cura di), 'Actio in rem'e 'actio in personam'. In ricordo di Mario Talamanca, II, Padova, 2011.

E. T. Salmon, Samnium and the Samnites, Cambridge 1967.

E. TodisCO, I 'vici’ rurali nel paesaggio dell'Italia romana, Bari 2011. 
E. TODISCO, La percezione delle realtà rurali nell'Italia romana: $i$ vici e i pagi, in M. PANI (ed.), Epigrafia e territorio, Bari 2004.

G. Franciosi, Osservazioni sulle strutture sociali dei Sanniti, in Opuscoli. Scritti di Gennaro Franciosi, Napoli 2012.

G. Tagliamonte, I Sanniti. Caudini, Irpini, Pentri ,Carricini , Frentani, Milano 1996.

G. ZARro, Aspetti dell'autonomia dei privati. Dalla fides ai nova negotia, Napoli 2015.

G. ZECCHINI, Il federalismo nel mondo antico, Milano 2005. H. FUGIER, Recherches sur l'expression du sacré dans la langue latine, Paris 1963.

J. LLOYD, Farming the highlands: Samnium and Arcadia in the Hellenistic and early Roman Imperial periods, in G. BARKER - A. LLOYD (cur.) Roman landscapes: archeological survey in the Mediterranean region, London 1991.

J. LLOYD, Pentri, frentani and the beginning of urbanization (500-80 BC), in G. BARKER (cur.) A Mediterranean valley: landscape archaeology and Annales history in the Biferno valley, Leicester 1995.

J. HeURGON, Recherches sur l'histoire, la religion et la civilisation de Capoue préromaine, Paris 1942.

K. J. BeLOCH, Campanien, Geschichte und Topographie des antiken Neapel und seiner Umgebung, Berlino, Calvary 1879; $2^{\mathrm{a}}$ ed., Breslavia, Morgenstern, 1890.

L. CAPOGROSSI COLOGNESI, 'Ius commerci' 'conubium' 'civitas sine suffragio'. Le origine del diritto internazionale privato e la romanizzazione delle comunità latino-campane, in L. CAPOGROSSI COLOGNESI-A. CORBINO-L. LABRUNA-B. SANTALUCIA, Le strade del potere, Catania 1994.

L. CAPogrossi Colognesi, Persistenza ed innovazione nelle strutture territoriali dell'Italia romana. L'ambiguità di una interpretazione storiografica e dei suoi modelli, Napoli 2002.

L. MonaCo, Brevi considerazioni in tema di strutture parentali dei Sanniti, in F. M.

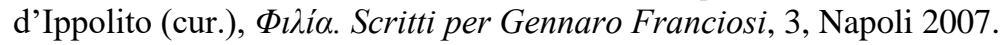

M. BetTini, Fas, in Giuristi Nati a cura di A. MCClintock, Bologna 2016.

N. D. Fustel De Coulanges, La cité antique. Etude sur le culte, le droit, les institutions de la Grèce et de Rome 1980, tr. it. 1924.

O. SACCHI, L'ager Campanus antiquus. Fattori di trasformazione e profili di storia giuridica del territorio dalla arcaica alla centuriatio romana, Napoli 2004.

P. CATALANo, Linee del sistema sovrannazionale romano, I, Torino 1965. 
P. NOAILlES, Du droit sacré au droit civil. Cours de droit romain approfondi 19411942, Paris 1949.

P. VocI, Rec. a Santoro, in Iura 34, 1983.

R. ORestano, Dal 'ius' al 'fas'. Rapporto fra diritto divino e umano in Roma dall'età primitiva all'età classica, in BIDR. 46, 1939.

R. ORESTANO, I fatti di normazione nell'esperienza romana arcaica, Torino 1967.

R. SANTORo, Potere ed azione nell'antico diritto romano, in Annali del Seminario giuridico dell'Università di Palermo 30, 1967.

R. SANTORO, Riflessioni sul contratto nel pensiero di Labeone, in Studi Arena 5, 1981.

R. SANTORO, Il contratto nel pensiero di Labeone, in AUPA. 37, 1983.

R. SANTORO, La causa nelle convenzioni atipiche, in Causa e contratto nella prospettiva storico comparatistica. II Congresso internazionale ARISTEC, Palermo-Trapani 1995.

R. SCHILLING, L'originalité du vocabulaire religieux latin, ora in ID., Rites, cultes, dieux de Rome, Paris 1979.

R. SCOPACASA, Ancient Samnium, Oxford 2015.

S. SISANI, In pagis forisque et conciliabulis. Le strutture amministrative dei distretti rurali in Italia tra la media Repubblica e l'età municipale, Roma 2011.

S. RANDAZZO, Lo statuto dello straniero e l'hospitium nel diritto romano arcaico, in Lo straniero e l'ospite. Diritto. Società. Cultura, Torino 2003.

T. J. CORNell, The Conquest of Italy, in CAH., VII.2², Cambridge 1989.

V. GIUFFRĖ, La "datio mutui": prospettive romane e moderne, Napoli 1989.

V. MAROTTA, Tutela dello scambio e commerci mediterranei in età arcaica e repubblicana, in Ostraka. Rivista di antichità 5, 1996. 
HAKEMSIZ MAKALELER 
